\title{
Partial Recursive Functions and Finality
}

\author{
Gordon Plotkin \\ LFCS, School of Informatics, University of Edinburgh
}

\begin{abstract}
We seek universal categorical conditions ensuring the representability of all partial recursive functions. In the category $\mathbf{P f n}$ of sets and partial functions, the natural numbers provide both an initial algebra and a final coalgebra for the functor $1+-$. We recount how finality yields closure of the partial functions on natural numbers under Kleene's $\mu$-recursion scheme. Noting that $\mathbf{P f n}$ is not cartesian, we then build on work of Paré and Román, obtaining weak initiality and finality conditions on natural numbers algebras in monoidal categories that ensure the (weak) representability of all partial recursive functions. We further obtain some positive results on strong representability. All these results adapt to Kleisli categories of cartesian categories with natural numbers algebras. However, in general, not all partial recursive functions need be strongly representable.
\end{abstract}

\section{Introduction}

It is a great pleasure to write in celebration of Samson Abramsky's 60th birthday. The interaction between category theory and computer science has long been central in Samson's work. Here we touch lightly on several themes of this kind which have been of interest to him: coalgebras, computability, definability, domain equations, linearity, and - evennatural numbers objects [11].

Our interest is in finding universal categorical conditions that ensure the representability of all partial recursive functions. The case of the primitive recursive functions is well understood. In general terms, the existence of a "natural numbers" algebra

$$
1 \longrightarrow \mathrm{N} \longleftarrow \mathrm{N}
$$

with sufficient initiality properties ensures the representability of all primitive recursive functions. More precisely, all primitive recursive functions are representable in any cartesian closed category with a weak natural numbers object, i.e., a weakly initial natural numbers algebra [7], and, more generally, in any cartesian category with a so-called weak stable natural numbers object $[7,2,10]$.

Natural numbers algebras can equivalently be written in the form

$$
1+\mathrm{N} \longrightarrow \mathrm{N}
$$

and it is then natural to consider the dual notion of natural numbers coalgebras

$$
\mathrm{N} \longrightarrow 1+\mathrm{N}
$$


and ask for final ones. As is known, for example from domain theory [5, Example IV-7.15], the natural numbers form a final such coalgebra in the category Pfn of sets and partial functions. We verify this directly in Section 3. We further recount there how finality leads to Kleene's $\mu$-recursion scheme.

One may therefore hope that all partial recursive functions are representable in any category possessing a natural numbers algebra with sufficiently strong initiality and finality properties. Such conditions should apply to Pfn, and, more broadly, to categories considered in general frameworks for partial functions, such as those of [3] or [4].

Unfortunately, $\mathbf{P f n}$ is not cartesian. Rather, cartesian product equips $\mathbf{P f n}$ with a symmetric monoidal structure. So the above work on representability in cartesian categories does not immediately apply. Instead, in Section 4, we turn to the work of Paré and Román [12]. They gave a notion of stable left (or right) natural numbers objects in monoidal categories that ensures the representability of all primitive recursive functions. Their proof uses the uniqueness clause of initiality. However we prefer to use only existence, as that is the common assumption used to ensure representability. This can be done using structural functions (such as symmetry) and, following Paré and Román, suitable versions of these can be defined over weak stable natural numbers objects. In [1] Alves et al considered the symmetric monoidal case using similar methods, but working in an informal type-theoretic setting rather than a categorical one.

Putting all this together in Section 5, we finally obtain universality properties that ensure the representability of all partial recursive functions (Theorem 1) and that can be applied to categories of partial functions. The notion of representability that we use is somewhat weak and there is a natural stronger one. We show that all total recursive functions, and, more generally, all partial recursive functions with recursive graphs, are strongly representable, under weak additional assumptions (Theorem 2). If we also assume that all definable partial functions are partial recursive, then all partial recursive functions are strongly representable (Corollary 1). However this fails in general, and we provide a syntactic category which has a stable natural numbers object which is also final but in which no partial recursive function with a non-recursive graph is strongly representable (Theorem 3).

For categorical terminology used below, the reader may refer, for example, to [2].

\section{Primitive recursive functions in cartesian categories}

We recall some definitions and results on natural numbers algebras in cartesian categories. Further information can be found in many places, for example $[7,2,10]$. We assume given a cartesian category $\mathbf{C}$, and a natural numbers algebra

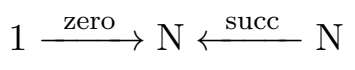


within it. We can then represent natural numbers and functions on them. First the numerals $\underline{k}: 1 \rightarrow \mathrm{N}($ for $k \in \mathbb{N})$ are defined by:

$$
\underline{k}=\operatorname{def} \operatorname{succ}^{k} \text { o zero }
$$

Then a morphism $g: \mathrm{N}^{n} \rightarrow \mathrm{N}$ is said to represent a function $f: \mathbb{N}^{n} \rightarrow \mathbb{N}$ if, for all $k_{1}, \ldots, k_{n} \in \mathbb{N}$ :

$$
g \circ\left\langle\underline{k_{1}}, \ldots, \underline{k_{n}}\right\rangle=\underline{f\left(k_{1}, \ldots, k_{n}\right)}
$$

The representable functions include some base functions: zero represents the constant 0 ; succ represents the successor function; and the projections $\pi_{i}: \mathbb{N}^{n} \rightarrow \mathbb{N}$, for $i=1, \ldots, n$ are also representable. The representable functions are also closed under composition, by which is meant that if $f: \mathbb{N}^{m} \rightarrow \mathbb{N}$ is representable, and so are $g_{i}: \mathbb{N}^{n} \rightarrow \mathbb{N}$, for $i=1, \ldots, m$, then $h: \mathbb{N}^{n} \rightarrow \mathbb{N}$ is representable, where, for $k_{1}, \ldots, k_{n} \in \mathbb{N}$ :

$$
h\left(k_{1}, \ldots, k_{n}\right)=_{\text {def }} f\left(g_{1}\left(k_{1}, \ldots, k_{n}\right), \ldots, g_{m}\left(k_{1},, k_{n}\right)\right)
$$

Our natural numbers algebra $1 \stackrel{\text { zero }}{\longrightarrow} \mathrm{N} \stackrel{\text { succ }}{\longleftarrow} \mathrm{N}$ is a natural numbers object if it is an initial natural numbers algebra, by which we mean that for any other such structure

$$
1 \stackrel{z}{\longrightarrow} B \stackrel{s}{\longleftarrow} B
$$

there is a unique map $h: \mathrm{N} \rightarrow B$ such that the following diagram commutes:

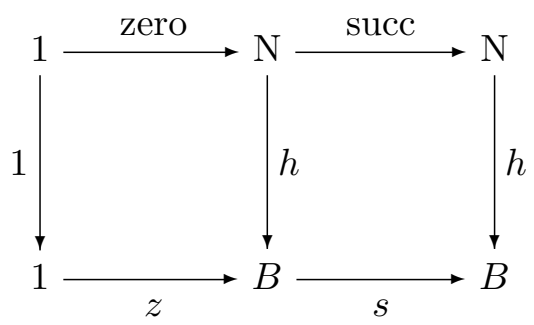

As an evident example, in the category Set of sets and (total) functions, the natural numbers algebra $\mathbb{1} \stackrel{\text { zero }}{\longrightarrow} \mathbb{N} \stackrel{\text { succ }}{\longleftarrow} \mathbb{N}$ is a natural numbers object, where zero $(*)={ }_{\text {def }} 0$ and $\operatorname{succ}(n)=_{\operatorname{def}} n+1$.

With initiality, the representable functions are closed under the following scheme, which, given $a \in \mathbb{N}$ and $g: \mathbb{N} \rightarrow \mathbb{N}$, yields $h: \mathbb{N} \rightarrow \mathbb{N}$ such that:

$$
\begin{aligned}
& h(0) \quad=a \\
& h(k+1)=g(h(k))
\end{aligned}
$$

However that is still too weak. Our natural numbers algebra $1 \stackrel{\text { zero }}{\longrightarrow} \mathrm{N} \stackrel{\text { succ }}{\longleftarrow} \mathrm{N}$ is a stable natural numbers object if, for any structure

$$
P \stackrel{f}{\longrightarrow} B \stackrel{g}{\longleftarrow} B
$$


there is a unique map $h: P \times \mathrm{N} \rightarrow B$ such that the following diagram commutes:

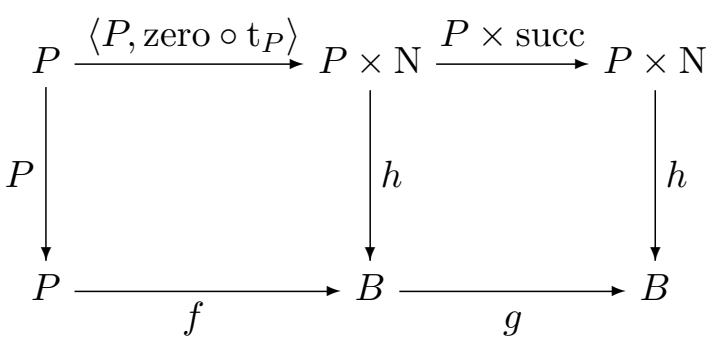

If $\mathbf{C}$ is cartesian closed then natural numbers objects are automatically stable, but that does not hold more generally. If we only have existence (but not uniqueness) we say that we have a weak stable natural numbers object.

With stability, indeed, even with weak stability, the representable functions are closed under pure iteration with $n$ parameters. This, given $f: \mathbb{N}^{n} \rightarrow \mathbb{N}$ and $g: \mathbb{N} \rightarrow \mathbb{N}$, yields $h: \mathbb{N}^{n+1} \rightarrow \mathbb{N}$ such that:

$$
\begin{array}{ll}
h\left(k_{1}, \ldots, k_{n}, 0\right) & =f\left(k_{1}, \ldots, k_{n}\right) \\
h\left(k_{1}, \ldots, k_{n}, k+1\right) & =g\left(h\left(k_{1}, \ldots, k_{n}, k\right)\right)
\end{array}
$$

By a result of Gladstone [6] we obtain all primitive recursive functions if we start from the base functions and close under composition and pure iteration (even allowing only one parameter).

In summary we have:

Proposition 1. All primitive recursive functions are representable in any cartesian category with a weak stable natural numbers object.

\section{The Category of Sets and Partial Functions}

We begin by reviewing some general ideas. Suppose we are in a category with a terminal object and binary sums. Then, as remarked in the introduction, natural numbers algebras $1 \stackrel{\text { zero }}{\longrightarrow} \mathrm{N} \stackrel{\text { succ }}{\longleftarrow} \mathrm{N}$ are in $1-1$ correspondence with $F$-algebras $(\mathrm{N}, \alpha: F(\mathrm{~N}) \rightarrow \mathrm{N})$ where $F$ is the endofunctor $F(A)={ }_{\operatorname{def}} 1+A$. This correspondence sends (zero, succ) to

$$
\alpha={ }_{\text {def }} 1+\mathrm{N} \stackrel{\text { [zero,succ] }}{\longrightarrow} \mathrm{N}
$$

(Weak) natural numbers objects correspond in this way to (weakly) initial $F$-algebras.

One can also discuss $F$-coalgebras of given endofunctors $F$. These are structures of the form $(A, \alpha: A \rightarrow F(A))$. In the case where $F=1+-$, we call such coalgebras natural numbers coalgebras. A homomorphism of $F$-coalgebras $h:(A, \alpha) \rightarrow(B, \beta)$ is a morphism 
$h: A \rightarrow B$ such that the following diagram commutes:

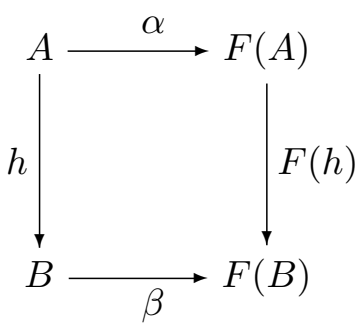

and one is interested in (weakly) final coalgebras.

A natural place to begin to explore these ideas is the category $\mathbf{P f n}$ of sets and partial functions, since partial recursive functions are morphisms there. We know that the natural numbers form an initial algebra $\alpha: F(\mathbb{N}) \cong \mathbb{N}$ in Set (the category of sets and total functions) where, as above, $F(X)=11+X$ and $\alpha(\operatorname{inl}(*))=0$ and $\alpha(\operatorname{inr}(n))=n+1$. As $\alpha$ is an isomorphism (as is, by Lambek's Lemma, the algebra map of the initial algebra of any endofunctor), we obtain a natural numbers coalgebra $\alpha^{-1}: \mathbb{N} \rightarrow F(\mathbb{N})$ in Set. Concretely one has:

$$
\alpha^{-1}(k)= \begin{cases}\operatorname{inl}(*) & (k=0) \\ \operatorname{inr}\left(k^{\prime}\right) & \left(k=k^{\prime}+1\right)\end{cases}
$$

This natural numbers coalgebra is not final in Set. However sums in Set are also sums in $\mathbf{P f n}$, and so $F$ extends to Pfn. As we now check, the coalgebra is final there.

We have to show that for any $\beta: Y \rightarrow \mathbb{1}+Y$ there is a unique map $h: Y \rightarrow \mathbb{N}$ such that the following diagram commutes in $\mathbf{P f n}$ :

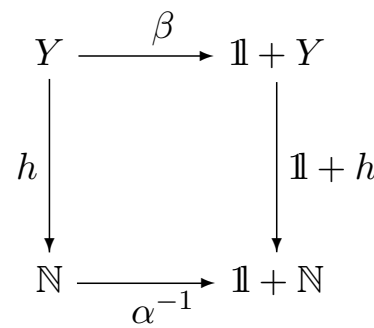

As $\alpha$ is an isomorphism this is equivalent to asking that the following diagram commutes:

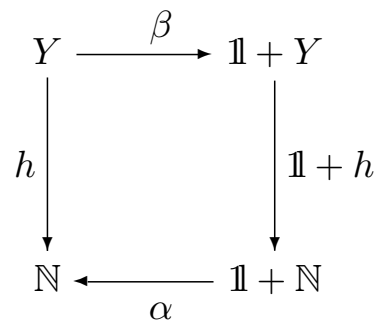


Using Kleene equality we can write this out as an equation:

$$
h(y) \simeq \begin{cases}0 & (\beta(y) \simeq \operatorname{inl}(*)) \\ h\left(y^{\prime}\right)+1 & \left(\beta(y) \simeq \operatorname{inr}\left(y^{\prime}\right)\right) \\ \text { undefined }(\beta(y) \uparrow)\end{cases}
$$

where, as usual, we write $e \uparrow$ to assert that an expression $e$ is undefined (and write $e \downarrow$ to assert it is defined).

One can show there is a unique such $h$. For uniqueness, for any $h, h^{\prime}$ satisfying the equation one proves by induction on $k$ that, for all $y, h(y) \simeq k$ if, and only if, $h^{\prime}(y) \simeq k$. For existence, one can set:

$$
h(y) \simeq_{\text {def }} \quad \mu k \in \mathbb{N} \cdot s^{k}(\beta(y)) \simeq \operatorname{inl}(*)
$$

where $s={ }_{\text {def }} \beta \circ$ inr $^{-1}$, and check $h$ satisfies (1). Here, as usual, $\mu k \in \mathbb{N} . \varphi(k)$ is the smallest $k \in \mathbb{N}$ such that $\varphi(k)$ holds, if there is one, and is undefined otherwise.

Given this connection with minimisation it may not now be surprising that we can obtain Kleene's $\mu$-recursion scheme from finality. Suppose we have $P: \mathbb{N}^{n+1} \rightarrow \mathbb{N}$. Then we apply finality to the coalgebra

$$
\beta: \mathbb{N}^{n+1} \longrightarrow \mathbb{1}+\mathbb{N}^{n+1}
$$

where

$$
\beta(\mathbf{x}, k) \simeq \begin{cases}\operatorname{inl}(*) & (P(\mathbf{x}, k) \simeq 0) \\ \operatorname{inr}(\mathbf{x}, k+1) & (P(\mathbf{x}, k) \downarrow \text { and } \neq 0) \\ \text { undefined } & (P(\mathbf{x}, k) \uparrow)\end{cases}
$$

Substituting into (1), we find that the unique $h: \mathbb{N}^{n+1} \rightarrow \mathbb{N}$ whose existence is guaranteed by finality satisfies the following equation:

$$
h(\mathbf{x}, k) \simeq \begin{cases}0 & (P(\mathbf{x}, k) \simeq 0) \\ h(\mathbf{x}, k+1)+1 & (P(\mathbf{x}, k) \downarrow \text { and } \not 40) \\ \text { undefined } & (P(\mathbf{x}, k) \uparrow)\end{cases}
$$

We then see that $h$ can be defined by the minimisation

$$
h(\mathbf{x}, k) \simeq \mu k^{\prime} \cdot P\left(\mathbf{x}, k+k^{\prime}\right) \simeq 0 \wedge \forall k^{\prime \prime}<k^{\prime} \cdot P\left(\mathbf{x}, k+k^{\prime \prime}\right) \downarrow
$$

as, with this definition, one checks that $h$ satisfies (2). Specialising, we obtain:

$$
h(\mathbf{x}, 0) \simeq \mu k \in \mathbb{N} \cdot P(\mathbf{x}, k) \simeq 0 \wedge \forall k^{\prime}<k \cdot P\left(\mathbf{x}, k^{\prime}\right) \downarrow
$$

and so $h(-, 0): \mathbb{N}^{n} \rightarrow \mathbb{N}$ is the partial function obtained by $\mu$-recursion from $P$, thereby establishing the advertised connection between finality and Kleene's $\mu$-recursion scheme. 


\section{Primitive Recursive Functions in Monoidal Categories}

We assume given a monoidal category $\mathbf{C}$ with the standard structural maps:

$$
\mathrm{a}_{A, B, C}: A \otimes(B \otimes C) \cong(A \otimes B) \otimes C \quad \mathrm{l}_{A}: \mathrm{I} \otimes A \cong A \quad \mathrm{r}_{A}: A \otimes \mathrm{I} \cong A
$$

satisfying the usual equations. We need some notation. For any object $A$ define $A^{n}$ by setting $A^{0}=\mathrm{I}$ and $A^{n+1}=A^{n} \otimes A$. For $n \geq 0$ and $c_{i}: \mathrm{I} \rightarrow A(i=1, \ldots, n)$, define $\left\langle c_{1}, \ldots, c_{n}\right\rangle: \mathrm{I} \rightarrow A^{n}$ by: \langle\rangle$=\operatorname{id}_{\mathrm{I}}$ and $\left\langle c_{1}, \ldots, c_{n}, c_{n+1}\right\rangle=\left(\left\langle c_{1}, \ldots, c_{n}\right\rangle \otimes c_{n+1}\right) \circ \mathrm{l}_{I}^{-1}$.

We next assume given a natural numbers algebra in $C$, by which we now mean a structure

$$
\mathrm{I} \stackrel{\text { zero }}{\longrightarrow} \mathrm{N} \stackrel{\text { succ }}{\longleftarrow} \mathrm{N}
$$

We define $\underline{k}: \mathrm{I} \rightarrow \mathrm{N}$ for $k \in \mathbb{N}$ to be succ ${ }^{k} \circ$ zero, and for any $\mathbf{k}=k_{1}, \ldots, k_{n}$ we write $\underline{\mathbf{k}}$ for $\left\langle k_{1}, \ldots, k_{n}\right\rangle: \mathrm{I} \rightarrow \mathrm{N}^{n}$. We then say that a morphism $f: \mathrm{N}^{n} \rightarrow \mathrm{N}$ represents a (total) function $f: \overline{\mathbb{N}^{n}} \rightarrow \mathbb{N}$ if, for all $k_{1}, \ldots, k_{n} \in \mathbb{N}$, we have:

$$
\underline{f} \circ\left\langle\underline{k_{1}}, \ldots, \underline{k_{n}}\right\rangle=\underline{f\left(k_{1}, \ldots, k_{n}\right)}
$$

For example, zero represents the constant 0 and succ represents the successor function.

The natural numbers algebra $\mathrm{I} \stackrel{\text { zero }}{\longrightarrow} \mathrm{N} \stackrel{\text { succ }}{\longleftarrow} \mathrm{N}$ is a right stable natural numbers object if, for any structure of the form

$$
P \stackrel{f}{\longrightarrow} B \stackrel{g}{\longleftarrow} B
$$

there is a unique morphism $h: P \otimes \mathrm{N} \rightarrow B$ such that the following diagram commutes:

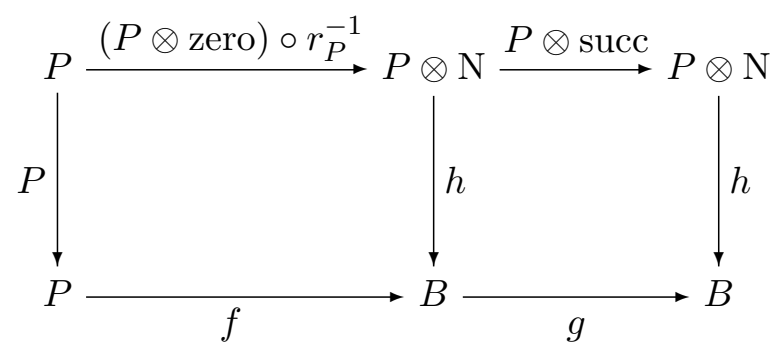

(and left stable natural numbers objects are defined symmetrically). As usual, one drops uniqueness for the weak notion. A (weak) right (or left) stable natural numbers object is a (weak) natural numbers object, in the evident sense.

From now on we assume our natural numbers algebra is a weak right stable natural numbers object.

Taking $P=\mathrm{N}^{n}$ in the definition of weak right stable natural numbers object we find that the morphism $h$ whose existence is asserted satisfies the following two equations:

$$
\begin{aligned}
& h \circ\left\langle\underline{k_{1}}, \ldots, \underline{k_{n}}, \underline{0}\right\rangle=f \circ\left\langle\underline{k_{1}}, \ldots, \underline{k_{n}}\right\rangle \\
& h \circ\left\langle\underline{k_{1}}, \ldots, \underline{k_{n}}, \underline{k+1}\right\rangle=g \circ h \circ\left\langle\underline{k_{1}}, \ldots, \underline{k_{n}}, \underline{k}\right\rangle
\end{aligned}
$$


So, in particular, taking $B=\mathrm{N}$ we find that the representable total functions on natural numbers are closed under the scheme of pure iteration with $n$ parameters. This, given $f: \mathbb{N}^{n} \rightarrow \mathbb{N}$ and $g: \mathbb{N} \rightarrow \mathbb{N}$, yields $h: \mathbb{N}^{n+1} \rightarrow \mathbb{N}$ such that:

$$
\begin{aligned}
& h\left(k_{1}, \ldots, k_{n}, 0\right)=f\left(k_{1}, \ldots, k_{n}\right) \\
& h\left(k_{1}, \ldots, k_{n}, k+1\right)=g\left(h\left(k_{1}, \ldots, k_{n}, k\right)\right)
\end{aligned}
$$

As we now show, weak stability can be used to obtain representations of structural maps involving $\mathbb{N}$, viz. terminal maps, projections, symmetries, and diagonals. First, considering $\mathrm{I} \stackrel{\mathrm{id}_{\mathrm{I}}}{\longrightarrow} \mathrm{I} \stackrel{\mathrm{id}_{\mathrm{I}}}{\longleftarrow} \mathrm{I}$ and applying weak initiality we obtain a morphism $\mathrm{t}_{\mathrm{N}}: \mathrm{N} \rightarrow \mathrm{I}$ such that:

$$
\begin{aligned}
& \mathrm{t}_{\mathrm{N}} \circ \underline{0}=\langle\rangle \\
& \mathrm{t}_{\mathrm{N}} \circ \underline{\underline{k}+1}=\mathrm{t}_{\mathrm{N}} \circ \underline{k}
\end{aligned}
$$

By induction on $k$, we then have $\mathrm{t}_{\mathrm{N}} \circ \underline{k}=\langle\rangle$. Using $\mathrm{t}_{\mathrm{N}}$, we obtain a morphism $\mathrm{t}_{\mathrm{N}^{n}}: \mathrm{N}^{n} \rightarrow \mathrm{I}$ such that $\mathrm{t}_{\mathrm{N}^{n}} \circ \underline{\mathbf{k}}=\langle\rangle$. Then, using $\mathrm{t}_{\mathrm{N}}$ and the $\mathrm{t}_{\mathrm{N}^{n}}$, we see that the projections $\pi_{i}^{n}: \mathbb{N}^{n} \rightarrow \mathbb{N}$ $(i=1, n)$ are representable. We also define $\pi_{1}: A \otimes \mathrm{N} \rightarrow A$ to be $\mathrm{r}_{A} \circ\left(A \otimes \mathrm{t}_{N}\right)$, and define $\pi_{2}: \mathrm{N} \otimes A \rightarrow A$ similarly; for any $k \in \mathbb{N}$ and $c: \mathrm{I} \rightarrow A$ we have $\pi_{1} \circ\langle c, \underline{k}\rangle=\pi_{2} \circ\langle\underline{k}, c\rangle=c$.

Regarding symmetry, considering $\mathrm{N} \stackrel{(\text { zero } \otimes \mathrm{N}) \circ \mathrm{o}_{\mathrm{N}}^{-1}}{\longrightarrow} \mathrm{N} \otimes \mathrm{N} \stackrel{\operatorname{succ} \otimes \mathrm{N}}{\longleftarrow} \mathrm{N} \otimes \mathrm{N}$ and applying weak stability, we obtain a morphism $\sigma_{\mathrm{N}, \mathrm{N}}: \mathrm{N} \otimes \mathrm{N} \rightarrow \mathrm{N} \otimes \mathrm{N}$ such that:

$$
\begin{aligned}
& \sigma_{\mathrm{N}} \circ\left(\underline{k_{1}} \otimes \underline{0}\right)=\underline{0} \otimes \underline{k_{1}} \\
& \sigma_{\mathrm{N}} \circ\left(\underline{k_{1}} \otimes \underline{k+1}\right)=(\operatorname{succ} \otimes \mathrm{N}) \circ \sigma_{\mathrm{N}} \circ\left(\underline{k_{1}} \otimes \underline{k}\right)
\end{aligned}
$$

and, by induction on $k$, we have $\sigma_{\mathrm{N}, \mathrm{N}} \circ\left(\underline{k_{1}} \otimes \underline{k}\right)=\left(\underline{k} \otimes \underline{k_{1}}\right)$. Using $\sigma_{\mathrm{N}, \mathrm{N}}$, we then obtain morphisms $\sigma_{\mathrm{N}^{m}, \mathrm{~N}^{n}}: \mathrm{N}^{m} \otimes \mathrm{N}^{n} \rightarrow \mathrm{N}^{n} \otimes \mathrm{N}^{m}$ such that $\sigma_{\mathrm{N}^{m}, \mathrm{~N}^{n}} \circ\left(\underline{\mathbf{k}} \otimes \underline{\mathbf{k}^{\prime}}\right)=\left(\underline{\mathbf{k}^{\prime}} \otimes \underline{\mathbf{k}}\right)$.

Regarding diagonal maps, considering $\mathrm{I} \stackrel{(\underline{0} \otimes \underline{0}) \mathrm{l}_{\mathrm{I}}^{-1}}{\longrightarrow} \mathrm{N} \otimes \mathrm{N} \stackrel{\text { succ } \otimes \text { succ }}{\longleftarrow} \mathrm{N} \otimes \mathrm{N}$ and applying weak initiality, we obtain a morphism $\Delta_{\mathrm{N}}: \mathrm{N} \rightarrow \mathrm{N} \otimes \mathrm{N}$ such that:

$$
\begin{aligned}
& \Delta_{\mathrm{N}} \circ \underline{0}=(\underline{0} \otimes \underline{0}) \circ \mathrm{l}_{\mathrm{I}}^{-1} \\
& \Delta_{\mathrm{N}} \circ \underline{k}+\underline{1}=(\operatorname{succ} \otimes \operatorname{succ}) \circ \Delta_{\mathrm{N}} \circ \underline{k}
\end{aligned}
$$

and then, by induction on $k$, we have $\Delta_{\mathrm{N}} \circ \underline{k}=(\underline{k} \otimes \underline{k}) \circ \mathrm{l}_{\mathrm{I}}^{-1}$. Using $\mathrm{t}_{N}$ and $\Delta_{\mathrm{N}}$ we then obtain diagonal morphisms $\Delta_{m}: \mathrm{N} \rightarrow \mathrm{N}^{m}(m \geq 0)$, and, in turn using the $\Delta_{m}$ and $\sigma$, we further obtain diagonal morphisms $\Delta_{n, m}: \mathrm{N}^{n} \rightarrow\left(\mathrm{N}^{n}\right)^{m}(m, n \geq 0)$ such that $\Delta_{n, m} \circ \underline{\mathbf{k}}=\langle\underline{\mathbf{k}}, \ldots, \underline{\mathbf{k}}\rangle$.

Finally, using the $\Delta_{n, m}$ and categorical composition, we see that the representable functions are closed under composition, i.e., that if $f: \mathbb{N}^{m} \rightarrow \mathbb{N}$ is representable, and so are $g_{i}: \mathbb{N}^{n} \rightarrow \mathbb{N}$, for $i=1, \ldots, m$, then $h: \mathbb{N}^{n} \rightarrow \mathbb{N}$ is representable, where, for $k_{1}, \ldots, k_{n} \in \mathbb{N}$ :

$$
h\left(k_{1}, \ldots, k_{n}\right)=_{\text {def }} f\left(g_{1}\left(k_{1}, \ldots, k_{n}\right), \ldots, g_{m}\left(k_{1}, \ldots, k_{n}\right)\right)
$$


For suppose that $f: \mathbb{N}^{m} \rightarrow \mathbb{N}$ is represented by the morphism $f: \mathrm{N}^{m} \rightarrow \mathrm{N}$ and that, for $i=1, \ldots, m, g_{i}: \mathbb{N}^{n} \rightarrow \mathbb{N}$ is represented by the morphism $g_{i}: \mathrm{N}^{n} \rightarrow \mathrm{N}$. Then their composition $h: \mathbb{N}^{n} \rightarrow \mathbb{N}$ is represented by the morphism $\underline{h}: \mathrm{N}^{n} \rightarrow \overline{\mathrm{N}}$, where

$$
\underline{h}==_{\text {def }} \quad \mathrm{N}^{n} \stackrel{\Delta_{n, m}}{\longrightarrow}\left(\mathrm{N}^{n}\right)^{m} \stackrel{\left(\ldots\left(\mathrm{I} \otimes \underline{g_{1}}\right) \otimes \ldots \otimes \underline{g_{m}}\right)}{\longrightarrow} \mathrm{N}^{m} \stackrel{\underline{f}}{\longrightarrow} \mathrm{N}
$$

By a result of Gladstone [6], all primitive recursive functions can be obtained starting from the base functions (zero, successor, and the projections) and closing under composition and pure iteration (even allowing only one parameter). We therefore have:

Proposition 2. All primitive recursive functions are representable in any monoidal category with a weak stable right (or left) natural numbers object.

(The case of a weak stable left natural numbers object follows by symmetry from that of a right one.)

\section{Partial Recursive Functions in Monoidal Categories}

We assume given a monoidal category $C$, as in the previous section, which also has binary sums. It would be natural to make a distributivity assumption. For example we might assume that the tensor right-distributes over binary sums, i.e., that the canonical map $B \otimes A+C \otimes A \rightarrow(B+C) \otimes A$ is an isomorphism. However, as with other structural maps, this proves unnecessary in the presence of weak stability.

Turning to natural numbers objects, we further assume given a natural numbers algebra

$$
\mathrm{I} \stackrel{\text { zero }}{\longrightarrow} \mathrm{N} \stackrel{\text { succ }}{\longleftarrow} \mathrm{N}
$$

which is a weak right stable natural numbers object such that the map

$$
\mathrm{I}+\mathrm{N} \stackrel{\alpha}{\longrightarrow} \mathrm{N}
$$

is an isomorphism, where $\alpha={ }_{\text {def }}$ [zero, succ], and is such that the coalgebra

$$
\mathrm{N} \stackrel{\alpha^{-1}}{\longrightarrow} \mathrm{I}+\mathrm{N}
$$

is weakly final.

The $\underline{k}$ are defined as above, but we need a wider notion of representability. Say that a morphism $g: \mathrm{N}^{n} \rightarrow \mathrm{N}$ represents a partial function $f: \mathbb{N}^{n} \rightarrow \mathbb{N}$ if, for all $k_{1}, \ldots, k_{n} \in \mathbb{N}$, if $f\left(k_{1}, \ldots, k_{n}\right) \simeq k$ then $g \circ\left\langle\underline{k_{1}}, \ldots, \underline{k_{n}}\right\rangle=\underline{k}$.

We know that all primitive recursive functions are representable, and one sees, much as in the total case, that the representable functions are closed under composition. So it remains to show that the representable functions are closed under Kleene's $\mu$-recursion scheme. 
We first need a weak version of right distributivity. Applying weak stability to the structure

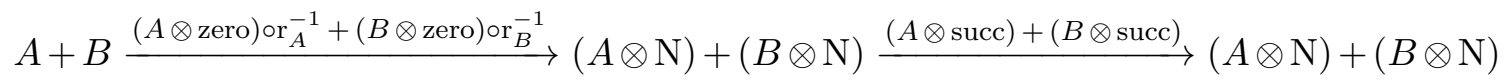

we obtain a map

$$
(A+B) \otimes \mathrm{N} \stackrel{\mathrm{d}_{A, B, N}}{\longrightarrow}(A \otimes \mathrm{N})+(B \otimes \mathrm{N})
$$

and, as can be shown by induction on $k$, for any $k$ and any $a: \mathrm{I} \rightarrow A$ and $b: \mathrm{I} \rightarrow B$, we have

$$
\mathrm{d}_{A, B, N} \circ((\operatorname{inl} \circ a) \otimes \underline{k})=\operatorname{inl} \circ(a \otimes \underline{k}) \quad \text { and } \quad \mathrm{d}_{A, B, N} \circ((\operatorname{inr} \circ b) \otimes \underline{k})=\operatorname{inr} \circ(b \otimes \underline{k})
$$

Iterating, for any $n$ we obtain a map:

$$
(A+B) \otimes \mathrm{N}^{n} \stackrel{\mathrm{d}_{A, B, N^{n}}}{\longrightarrow}\left(A \otimes \mathrm{N}^{n}\right)+\left(B \otimes \mathrm{N}^{n}\right)
$$

such that for any $k_{1}, \ldots, k_{n}$ and any $a: \mathrm{I} \rightarrow A$ and $b: \mathrm{I} \rightarrow B$, we have

and

$$
\mathrm{d}_{A, B, N} \circ\left((\operatorname{inl} \circ a) \otimes\left\langle\underline{k_{1}}, \ldots, \underline{k_{n}}\right\rangle\right)=\operatorname{inl} \circ\left(a \otimes\left\langle\underline{k_{1}}, \ldots, \underline{k_{n}}\right\rangle\right)
$$

$$
\mathrm{d}_{A, B, N} \circ\left((\operatorname{inr} \circ b) \otimes\left\langle\underline{k_{1}}, \ldots, \underline{k_{n}}\right\rangle\right)=\operatorname{inr} \circ\left(b \otimes\left\langle\underline{k_{1}}, \ldots, \underline{k_{n}}\right\rangle\right)
$$

Spelling out weak finality, we have that for any coalgebra $\beta: B \rightarrow \mathrm{I}+B$ there is a morphism $h: B \rightarrow \mathrm{N}$ such that (equivalently) either of the following two diagrams commute:
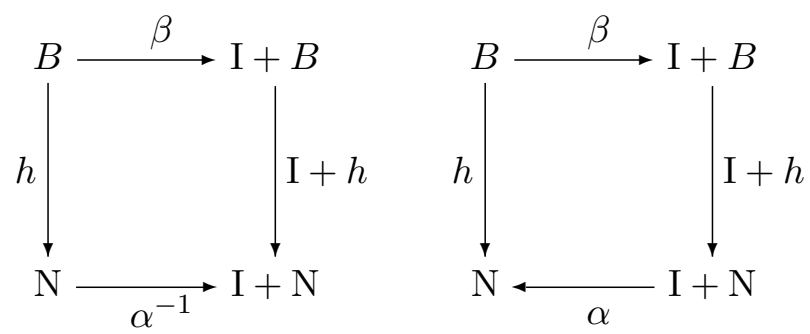

Looking at the second diagram, we obtain two equations, holding for any $b, b^{\prime}: \mathrm{I} \rightarrow B$ :

$$
\begin{gathered}
h \circ b=\text { zero } \quad(\text { if } \beta \circ b=\text { inl }) \\
h \circ b=\operatorname{succ} \circ h \circ b^{\prime} \quad\left(\text { if } \beta \circ b=\text { inr } \circ b^{\prime}\right)
\end{gathered}
$$

Now, to show closure under $\mu$-recursion, suppose $P: \mathbb{N}^{n} \times \mathbb{N} \rightarrow \mathbb{N}$ is represented by a morphism $\underline{P}: \mathrm{N}^{n} \otimes \mathrm{N} \rightarrow \mathrm{N}$. Then we apply weak finality to the coalgebra

$$
\begin{array}{cc}
\mathrm{N}^{n+1} & \stackrel{\left(\mathrm{N}^{n+1}\right)^{2}}{\stackrel{\Delta_{n+1,2}}{\longrightarrow}} \\
\stackrel{\left(\alpha^{-1} \circ \underline{P} \circ \mathrm{I}_{\mathrm{N}^{n+1}}\right) \otimes \mathrm{N}^{n+1}}{\longrightarrow} & (\mathrm{I}+\mathrm{N}) \otimes \mathrm{N}^{n+1} \\
\stackrel{\left[\pi_{1},\left(\mathrm{~N}^{n} \otimes \mathrm{succ}\right) \circ \pi_{2}\right]}{\longrightarrow} & \left(\mathrm{I} \otimes \mathrm{N}^{n+1}\right)+\left(\mathrm{N} \otimes \mathrm{N}^{n+1}\right) \\
& \mathrm{I}+\mathrm{N}^{n+1}
\end{array}
$$


and obtain a morphism

$$
h: \mathrm{N}^{n+1} \rightarrow \mathrm{N}
$$

obeying the following two equations:

$$
\begin{array}{ll}
h \circ\left\langle\underline{k_{1}}, \ldots, \underline{k_{n}}, \underline{k}\right\rangle=\text { zero } & \text { (if } \underline{P} \circ\left\langle\underline{k_{1}}, \ldots, \underline{k_{n}}, \underline{k}\right\rangle=\text { zero) } \\
h \circ\left\langle\underline{k_{1}}, \ldots, \underline{k_{n}}, \underline{k}\right\rangle=\operatorname{succ} \circ h \circ\left\langle\underline{k_{1}}, \ldots, \underline{k_{n}}, \underline{k+1}\right\rangle \quad & \text { (if } \underline{P} \circ\left\langle\underline{k_{1}}, \ldots, \underline{k_{n}}, \underline{k}\right\rangle=\operatorname{succ} \circ \underline{k_{k}^{\prime}}, \\
\left.\quad \text { for some } k^{\prime}\right)
\end{array}
$$

One then proves by induction on $l$ that, for all $k_{1}, \ldots, k_{n}, k$, we have:

$$
\mu k^{\prime} .\left(P\left(k_{1}, \ldots, k_{n}, k+k^{\prime}\right) \simeq 0 \wedge \forall k^{\prime \prime}<k^{\prime} . P\left(\mathbf{x}, k+k^{\prime \prime}\right) \downarrow\right) \simeq l \quad \Rightarrow \quad h \circ\left\langle\underline{k_{1}}, \ldots, \underline{k_{n}}, \underline{k}\right\rangle=\underline{l}
$$

and so we see that $h$ represents the partial function

$$
f\left(k_{1}, \ldots, k_{n}, k\right) \simeq_{\text {def }} \quad \mu k^{\prime} . P\left(k_{1}, \ldots, k_{n}, k+k^{\prime}\right) \simeq 0 \wedge \forall k^{\prime \prime}<k^{\prime} . P\left(\mathbf{x}, k+k^{\prime \prime}\right) \downarrow
$$

We therefore have, as required, that the representable functions are closed under Kleene's $\mu$-recursion scheme, as $h \circ\left(\mathrm{N}^{k} \otimes\right.$ zero $) \circ \mathrm{r}_{\mathrm{N}^{n}}^{-1}$ represents the partial function

$$
g\left(k_{1}, \ldots, k_{n}\right) \simeq_{\text {def }} \quad \mu k . P\left(k_{1}, \ldots, k_{n}, k\right) \simeq 0 \wedge \forall k^{\prime}<k . P\left(\mathbf{x}, k^{\prime}\right) \downarrow
$$

Our discussion has established:

Theorem 1. Let $\mathbf{C}$ be a monoidal category with binary sums and a weak left (or right) natural numbers object $\mathrm{I} \stackrel{\text { zero }}{\longrightarrow} \mathrm{N} \stackrel{\text { succ }}{\longleftarrow} \mathrm{N}$ such that [zero, succ] is an isomorphism and $\left(\mathrm{N},[\text { zero, succ }]^{-1}\right)$ is a weakly final natural numbers coalgebra. Then all partial recursive functions are representable in $\mathbf{C}$.

There is a natural stronger notion of representability of partial functions over $\mathbb{N}$ which we now investigate. Say that a morphism $g: \mathrm{N}^{n} \rightarrow \mathrm{N}$ strongly represents a partial function $f: \mathbb{N}^{n} \rightarrow \mathbb{N}$ if, for all $k_{1}, \ldots, k_{n} \in \mathbb{N}, f\left(k_{1}, \ldots, k_{n}\right) \simeq k$ if, and only if, $g \circ\left\langle\underline{k_{1}}, \ldots, \underline{k_{n}}\right\rangle=\underline{k}$.

Lemma 1. Let $\mathbf{C}$ be a monoidal category with a weak left (or right) natural numbers object $\mathrm{I} \stackrel{\text { zero }}{\longrightarrow} \mathrm{N} \stackrel{\text { succ }}{\longleftarrow} \mathrm{N}$ such that $\underline{0} \neq \underline{1}$. Then all representable total functions are strongly representable.

Proof. We first remark that if $\underline{k}=\underline{k^{\prime}}$ then $k=k^{\prime}$. For if not, as the predecessor function is representable, we get $\underline{0}=\underline{1}$, contradicting our assumption.

Now, suppose that $g: \mathrm{N}^{n} \rightarrow \mathrm{N}$ represents a total function $f: \mathbb{N}^{n} \rightarrow \mathbb{N}$, and choose $k_{1}, \ldots, k_{n}$. Then $g \circ\left\langle\underline{k_{1}}, \ldots, \underline{k_{n}}\right\rangle=f\left(k_{1}, \ldots, k_{n}\right)$. So if $g \circ\left\langle\underline{k_{1}}, \ldots, \underline{k_{n}}\right\rangle=\underline{k}$ then, by the remark, we have, as required, $f\left(k_{1}, \ldots, k_{n}\right)=k$.

Theorem 2. Let $\mathbf{C}$ be a monoidal category with binary sums and a weak left (or right) natural numbers object $\mathrm{I} \stackrel{\text { zero }}{\longrightarrow} \mathrm{N} \stackrel{\text { succ }}{\longleftarrow} \mathrm{N}$ such that [zero, succ] is an isomorphism and $\left(\mathrm{N},[\text { zero, succ }]^{-1}\right)$ is a weakly final natural numbers coalgebra. Then: 
1. If $\underline{0} \neq \underline{1}$ then all total recursive functions are strongly representable in $\mathbf{C}$.

2. If succ $\circ c \neq$ zero for all $c: \mathrm{I} \rightarrow \mathrm{N}$, then all partial recursive functions with recursive graphs are strongly representable in $\mathbf{C}$.

Proof. The first part follows immediately from Theorem 1 and Lemma 1. For the second part, suppose that $g: \mathbb{N}^{n} \rightarrow \mathbb{N}$ has a recursive graph. Then there is a recursive function $P: \mathbb{N}^{n+1} \rightarrow \mathbb{N}$ such that $P\left(\mathbf{k}, k^{\prime}\right)$ holds iff $g(\mathbf{k}) \simeq k^{\prime}$. Let $\underline{P}$ represent $P$, and define $h: \mathrm{N}^{n+1} \rightarrow \mathrm{N}$ and $f: \mathbb{N}^{n+1} \rightarrow \mathbb{N}$ as in the above derivation of $\mu$-recursion from weak finality. Then $h \circ\left(\mathrm{N}^{k} \otimes\right.$ zero $) \circ \mathrm{r}_{\mathrm{N}^{n}}^{-1}$ represents $g$. To show the representation is strong, it suffices to show that $h$ strongly represents $f$ (we already know it represents $f$ ).

To that end, fixing $\mathbf{k}$, we show, by course-of-values induction on $l$, that, for all $k^{\prime}$, if $h \circ\left\langle\underline{\mathbf{k}}, \underline{k}^{\prime}\right\rangle=\underline{l}$ then $f\left(\mathbf{k}, k^{\prime}\right) \simeq l$. Suppose, first, that $P\left(\mathbf{k}, k^{\prime}\right) \simeq 0$. Then we have $f\left(\mathbf{k}, k^{\prime}\right) \simeq 0$ and, using (3), that $\underline{l}=h \circ\left\langle\underline{\mathbf{k}}, \underline{k^{\prime}}\right\rangle=\underline{0}$. Then, by the assumption, we have $l=0$ and this case concludes.

Otherwise we have $P\left(\mathbf{k}, k^{\prime}\right) \downarrow$ and $\neq 0$ and then we have $f\left(\mathbf{k}, k^{\prime}\right) \simeq f\left(\mathbf{k}, k^{\prime}+1\right)+1$ and, using (4), that $\underline{l}=h \circ\left\langle\underline{\mathbf{k}}, \underline{k^{\prime}}\right\rangle=\operatorname{succ} \circ h \circ\left\langle\underline{\mathbf{k}}, \underline{k^{\prime}+1}\right\rangle$. Hence, by the assumption, we have $l \neq 0$, and we can apply the induction hypothesis, as succ has a left inverse.

The strong representability of all partial recursive functions can be established under a further, computability, assumption. Assuming that $\underline{0} \neq \underline{1}$ holds in our given category, every morphism $g: \mathrm{N}^{n} \rightarrow \mathrm{N}$ can be seen as defining a partial function $\bar{g}: \mathbb{N}^{n} \rightarrow \mathbb{N}$, where

$$
\bar{g}\left(k_{1}, \ldots, k_{n}\right) \simeq k \equiv_{\text {def }} g \circ\left\langle\underline{k_{1}}, \ldots, \underline{k_{n}}\right\rangle \simeq \underline{k}
$$

Note that $g$ strongly represents $\bar{g}$. We have:

Corollary 1. Let $\mathbf{C}$ be a monoidal category with binary sums and a weak left (or right) natural numbers object I $\stackrel{\text { zero }}{\longrightarrow} \mathrm{N} \stackrel{\text { succ }}{\longleftarrow} \mathrm{N}$ such that [zero, succ] is an isomorphism and $\left(\mathrm{N},[\text { zero, succ }]^{-1}\right)$ is a weakly final natural numbers coalgebra.

Then, if $\underline{0} \neq \underline{1}$ and if all strongly representable partial functions are partial recursive, all partial recursive functions are strongly representable.

Proof. A theorem of Visser [14, III.7] states that any class of unary partial recursive functions that (1) contains an upper bound of every partial recursive function and (2) is closed under right composition with all total recursive functions consists of all unary partial recursive functions.

Consider the class of all strongly representable (equivalently definable) unary partial functions. By assumption, these are all partial recursive. By Theorem 1, the first of the two conditions hold. By Theorem 2 every total recursive function is strongly representable and it is easy to see that the class of strongly representable unary partial functions is closed under right composition with strongly representable unary total functions. So the second condition also holds and Visser's theorem applies, showing that all unary partial recursive functions are strongly representable. It follows easily that all $n$-ary partial recursive functions are strongly representable. 
The corollary applies to various free categories such as the free monoidal category of the kind assumed given in this section.

These results can be applied to Kleisli categories. Suppose we have a cartesian category C with binary sums and a commutative strong monad T. Then the cartesian structure of $\mathbf{C}$ induces a symmetric monoidal structure on the Kleisli category $\mathbf{C}_{\mathrm{T}}[8,13]$. Further, $\mathbf{C}_{\mathrm{T}}$ inherits binary sums from C. As we are now in a symmetric situation, there is no need to distinguish between left and right (weak) natural numbers objects; so assume next that $1 \stackrel{\text { zero }}{\longrightarrow} \mathrm{N} \stackrel{\text { succ }}{\longleftarrow} \mathrm{N}$ is a (weak) stable natural numbers object in $\mathbf{C}$. One can check that it is also a (weak) stable numbers object in $\mathbf{C}_{\mathrm{T}}$ (more precisely that $1 \stackrel{\eta_{\mathrm{N}} \text { o zero }}{\longrightarrow} \mathrm{N} \stackrel{\eta_{\mathrm{N}} \text { o succ }}{\longleftarrow} \mathrm{N}$ is). So, if we further assume that $\left[\eta_{\mathrm{N}} \circ\right.$ zero, $\left.\eta_{\mathrm{N}} \circ \mathrm{succ}\right]$ is an isomorphism whose inverse provides a weak final natural numbers coalgebra in $\mathbf{C}_{\mathrm{T}}$, then all the above general results apply to $\mathbf{C}_{\mathrm{T}}$.

In $[3,4]$ categories of partial functions are seen as Kleisli categories for so-called "lifting" monads on cartesian categories. As an example, in any distributive category, -+1 is an equational lifting monad in the sense of [3]. As lifting monads are commutative, the discussion of Kleisli categories applies to them.

We know from Theorem 2 that, under a weak condition, all partial recursive functions with recursive graphs are strongly representable. We now see that this need not be the case if the graphs are not recursive. We first show how, given a consistent extension $\mathbf{T}$ of Peano arithmetic, to construct a distributive category $\mathbf{C}$ containing a stably initial natural numbers object which also provides a final coalgebra in the Kleisli category of the lifting monad -+1 . The desired counterexample is then obtained by a suitable choice of $\mathbf{T}$.

So let $\mathbf{T}$ be a consistent extension of Peano arithmetic. We allow ourselves to employ symbols for primitive recursive functions and assume their recursive definitions available in $\mathbf{T}$, and make use of evident multifix notation for them.

Fix three distinct variables $z, x$ and $y$. Given formulas $\varphi(z)$ and $\psi(z)$ whose only possible free variable is $z$, and a formula $\gamma(x, y)$ whose only possible free variables are $x$ and $y$, say that $\gamma$ is a $\mathbf{T}$-relation from $\varphi$ to $\psi$ if:

$$
\vdash_{\mathbf{T}} \varphi(x) \wedge \gamma(x, y) \Rightarrow \psi(y)
$$

that it is $\mathbf{T}$-function from $\varphi$ to $\psi$ if, in addition:

$$
\vdash_{\mathbf{T}} \varphi(x) \wedge \gamma(x, y) \wedge \gamma\left(x, y^{\prime}\right) \Rightarrow y=y^{\prime}
$$

and that it is a total $\mathbf{T}$-function from $\varphi$ to $\psi$ if, further:

$$
\vdash_{\mathbf{T}} \varphi(x) \Rightarrow \exists y \cdot \gamma(x, y)
$$

Define an equivalence relation on $\mathbf{T}$-relations from $\varphi$ to $\psi$ by:

$$
\gamma \sim \gamma^{\prime} \equiv \vdash_{\mathbf{T}} \varphi(x) \Rightarrow\left(\gamma(x, y) \Leftrightarrow \gamma^{\prime}(x, y)\right)
$$


Note that if $\gamma \sim \gamma^{\prime}$ then $\gamma$ is a (total) T-function from $\varphi$ to $\psi$ if, and only if $\gamma^{\prime}$ is.

The objects of $\mathbf{p C}$ are the formulas $\varphi$ whose only possible free variable is $z$ and the morphisms $[\gamma]: \varphi \rightarrow \psi$ of $\mathbf{p C}$ are the $\sim$-equivalence classes of $\mathbf{T}$-functions $\gamma$ from $\varphi$ to $\psi$. Identities and composition are given by:

$$
\varphi \stackrel{\operatorname{id}_{\varphi}}{\longrightarrow} \varphi=[y=x]
$$

and

$$
[\delta] \circ[\gamma]=[\exists w \cdot \gamma(x, w) \wedge \delta(w, y)]
$$

where $[\gamma]: \varphi \rightarrow \psi$ and $\delta: \psi \rightarrow \chi$.

The total $\mathbf{T}$-functions form a subcategory $\mathbf{C}$ of $\mathbf{p C}$ which we now investigate. It is distributive. The final object is $z=0$ with $\varphi \stackrel{\mathrm{t}}{\rightarrow} 1=[y=0]$. Binary products are given by $\varphi \times \psi=\varphi\left(\pi_{1}(z)\right) \wedge \psi\left(\pi_{2}(z)\right)$, with projections $\pi_{i}=\left[y=\pi_{i}(x)\right](i=1,2)$ and with $\langle\gamma, \delta\rangle=\left[\gamma\left(x, \pi_{1}(z)\right) \wedge \delta\left(x, \pi_{2}(z)\right)\right]$, for $[\gamma]: \chi \rightarrow \varphi,[\delta]: \chi \rightarrow \psi$ (we make use of a surjective pairing function).

The initial object is $\perp$ with $0 \stackrel{\mathrm{i}}{\rightarrow} \varphi=[\perp]$. Binary sums are given by

$$
\varphi+\psi=(\exists w . z=2 w \wedge \varphi(w)) \vee(\exists w . z=2 w+1 \wedge \psi(w))
$$

with coprojections inl $=[y=2 x]$ and inr $=[y=2 x+1]$ and with

$$
[[\gamma],[\delta]]=[(\exists w \cdot x=2 w \wedge \gamma(w, y)) \vee(\exists w \cdot x=2 w+1 \wedge \delta(w, y))]
$$

for $[\gamma]: \varphi \rightarrow \chi,[\delta]: \psi \rightarrow \chi$. It is not hard to see that products distribute over sums.

Next, we have a natural numbers algebra

$$
1 \stackrel{\text { zero }}{\longrightarrow} \mathrm{N} \stackrel{\text { succ }}{\longleftarrow} \mathrm{N}
$$

where $\mathrm{N}=\mathrm{T}$, zero $=_{\operatorname{def}}[y=0]$, and $\operatorname{succ}=_{\operatorname{def}}[y=\mathrm{s}(x)]$. Considered as an algebra $\alpha: 1+\mathrm{N} \rightarrow \mathrm{N}$, we have:

$$
\alpha=[(x=0 \wedge y=0) \vee(\exists w \cdot x=2 w+1 \wedge y=\operatorname{succ}(w))]
$$

Note that $\underline{k}=\left[y=\mathrm{s}^{k}(0)\right]$. Also, succ $\circ c \neq$ zero, for all $c: 1 \rightarrow \mathrm{N}$, as $\mathbf{T}$ is a consistent extension of Peano arithmetic.

We next check that our natural numbers algebra is a stable natural numbers object. As $\mathbf{C}$ is cartesian, it is enough to show that for any structure of the form

$$
\varphi \stackrel{[\gamma]}{\longrightarrow} \psi \stackrel{[\delta]}{\longleftarrow} \psi
$$

there is a unique $[\theta]: \varphi \times \mathrm{N} \rightarrow \psi$ such that the following diagram commutes: 


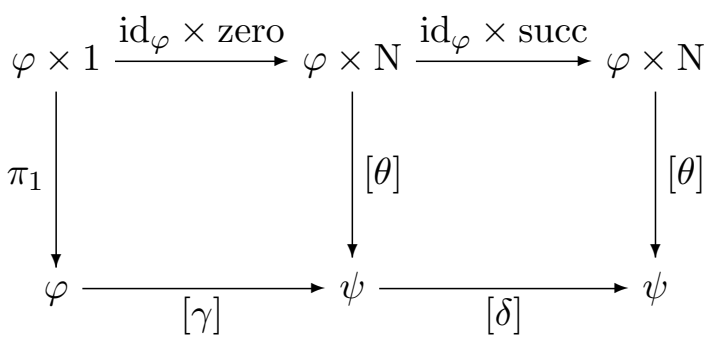

The diagram commutes if, and only if both

$$
\vdash_{\mathbf{T}} \varphi\left(x_{1}\right) \Rightarrow\left(\theta\left(\left\langle x_{1}, 0\right\rangle, y\right) \Leftrightarrow \gamma\left(x_{1}, y\right)\right)
$$

and

$$
\vdash_{\mathbf{T}} \varphi\left(x_{1}\right) \Rightarrow\left(\theta\left(\left\langle x_{1}, \mathrm{~s}\left(x_{2}\right)\right\rangle, y\right) \Leftrightarrow \exists w \cdot \theta\left(\left\langle x_{1}, x_{2}\right\rangle, w\right) \wedge \delta(w, y)\right)
$$

hold. For uniqueness, given $\theta$ and $\theta^{\prime}$ satisfying these two conditions, one shows that

$$
\vdash_{\mathbf{T}} \varphi\left(x_{1}\right) \Rightarrow\left(\theta\left(\left\langle x_{1}, x_{2}\right\rangle, y\right) \Leftrightarrow \theta^{\prime}\left(\left\langle x_{1}, x_{2}\right\rangle, y\right)\right)
$$

holds, using induction on $x_{2}$. For existence, one defines $\theta$ using codes for sequences, following the standard method used to show that the primitive recursive functions are representable in Peano arithmetic.

We next interest ourselves in the Kleisli category $\mathbf{C}_{L}$, where $L={ }_{\text {def }}-+1$ is the equational lifting monad available in any distributive category. By a remark made above, $1 \stackrel{\text { zero }}{\longrightarrow} \mathrm{N} \stackrel{\text { succ }}{\longleftarrow} \mathrm{N}$ is a stable natural numbers object in $\mathbf{C}_{L}$ as it is in $\mathbf{C}$. The $\underline{k}$ in $\mathbf{C}_{L}$ are as in $\mathbf{C}$, but composed with the unit $\eta_{\mathrm{N}}: \mathrm{N} \rightarrow \mathrm{N}+1$. As $\eta_{\mathrm{N}}$ has a left inverse, the condition that succ $\circ c \neq$ zero for all $c: 1 \rightarrow \mathrm{N}$ is inherited by $\mathbf{C}_{L}$ from $\mathbf{C}$.

We wish to check next that $\left(\mathrm{N},\left[\eta_{\mathrm{N}} \circ \text { zero, } \eta_{\mathrm{N}} \circ \mathrm{succ}\right]^{-1}\right)$ is a final $(1+-)$-coalgebra in the Kleisli category $\mathbf{C}_{L}$. There is an equivalence of categories

$$
F \dashv G: \mathbf{C}_{L} \cong \mathbf{p C}
$$

where both $F$ and $G$ are the identity on objects,

$$
F([\gamma])=[(\exists w \cdot \gamma(x, w) \wedge y=2 w) \vee(\neg \exists w \cdot \gamma(x, w) \wedge y=1)]
$$

for $[\gamma]: \varphi \rightarrow \psi$ in $\mathbf{p C}$ and

$$
G([\delta])=[\delta(x, 2 y)]
$$

for $[\delta]: \varphi \rightarrow \psi$ in $\mathbf{C}_{L}$. Under this equivalence (N, $\left.\left[\eta_{\mathrm{N}} \circ \text { zero, } \eta_{\mathrm{N}} \circ \mathrm{succ}\right]^{-1}\right)$ is a final $(1+-)-$ coalgebra in $\mathbf{C}_{L}$ if, and only if, (N, [zero, succ $]^{-1}$ ) is in $\mathbf{p C}$.

We therefore now check that the latter is such a coalgebra, guided by the corresponding discussion in Section 3. Noting that, in pC, every object is a retract of N, it is enough to 
check that for any coalgebra $[\gamma]: \mathrm{N} \rightarrow 1+\mathrm{N}$ there is a unique morphism $[\delta]: \mathrm{N} \rightarrow \mathrm{N}$ such that the following diagram commutes:

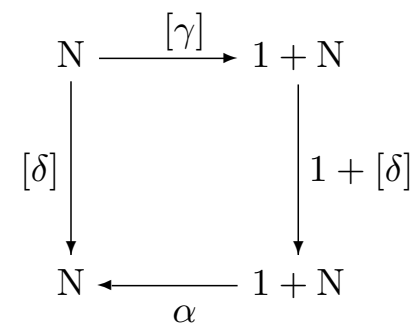

This diagram commutes if, and only if, the following holds:

$$
\vdash_{\mathbf{T}} \delta(x, y) \Leftrightarrow \exists v \cdot \gamma(x, v) \wedge\left[(v=0 \wedge y=0) \vee\left(\exists v^{\prime} \cdot v=2 v^{\prime}+1 \wedge \exists w \cdot \delta\left(v^{\prime}, w\right) \wedge y=\mathrm{s}(w)\right)\right]
$$

equivalently if, and only if, both

$$
\vdash_{\mathbf{T}} \delta(x, 0) \Leftrightarrow \gamma(x, 0)
$$

and

$$
\vdash_{\mathbf{T}} \delta(x, \mathrm{~s}(y)) \Leftrightarrow \exists w \cdot \gamma(x, \mathrm{~s}(w)) \wedge \delta(w, y)
$$

hold.

The uniqueness of $\delta$, up to $\sim$, is shown by induction on $y$. For its existence, we first define $[\theta]: \mathrm{N} \times \mathrm{N} \rightarrow \mathrm{N}$ by weak stability so that

$$
\vdash_{\mathbf{T}} \theta\left(\left\langle x_{1}, 0\right\rangle, y\right) \Leftrightarrow \gamma\left(x_{1}, y\right) \text { and } \vdash_{\mathbf{T}} \theta\left(\left\langle x_{1}, \mathrm{~s}\left(x_{2}\right)\right\rangle, y\right) \Leftrightarrow \exists w \cdot \theta\left(\left\langle x_{1}, x_{2}\right\rangle, \mathrm{s}(w)\right) \wedge \gamma(w, y)
$$

and then set:

$$
\delta(x, y)=_{\operatorname{def}} \theta(\langle x, y\rangle, 0) \wedge \forall y^{\prime}<y \cdot \neg \theta\left(\left\langle x, y^{\prime}\right\rangle, 0\right)
$$

The formula $\delta(x, y)$ is evidently $\mathbf{T}$-functional and it evidently satisfies (5). To show it satisfies (6), one first shows that

$$
\vdash_{\mathbf{T}} \theta\left(\left\langle x_{1}, s\left(x_{2}\right)\right\rangle, y\right) \Leftrightarrow \exists w \cdot \gamma\left(x_{1}, \mathrm{~s}(w)\right) \wedge \theta\left(\left\langle w, x_{2}\right\rangle, y\right)
$$

holds, using induction on $x_{2}$. Using (7), one then shows that

$$
\vdash_{\mathbf{T}} \gamma\left(x_{1}, \mathrm{~s}(w)\right) \Rightarrow\left[\left(\forall y^{\prime}<\mathrm{s}(y) . \neg \theta\left(\left\langle x_{1}, y^{\prime}\right\rangle, 0\right)\right) \Leftrightarrow\left(\forall y^{\prime}<y \cdot \neg \theta\left(\left\langle w, y^{\prime}\right\rangle, 0\right)\right)\right]
$$

holds. Finally, one proves $\delta(x, y)$ satisfies (6) by using its definition and then (7) and (8).

A formula $\chi\left(x_{1}, \ldots, x_{n}\right)$ semi-represents a relation $R \subseteq \mathbb{N}^{n}$ in an extension $\mathbf{T}$ of Peano arithmetic, if, for all $k_{1}, \ldots, k_{n}, R\left(k_{1}, \ldots, k_{n}\right)$ holds if, and only if, $\vdash_{\mathbf{T}} \chi\left(\underline{k_{1}}, \ldots, \underline{k_{n}}\right)$ does. 
Theorem 3. There is a distributive category $\mathbf{C}$ with a stable natural numbers object

$$
1 \stackrel{\text { zero }}{\longrightarrow} \mathrm{N} \stackrel{\text { succ }}{\longleftarrow} \mathrm{N}
$$

such that in the Kleisli category $\mathbf{C}_{L}$, where $L=(-+1)$ :

1. $\left(\mathrm{N},[\text { zero, }, \mathrm{succ}]^{-1}\right)$ is a final natural numbers coalgebra, and

2. succ $\circ c \neq$ zero, for all $c: 1 \rightarrow \mathrm{N}$, but

3. the only strongly representable partial recursive functions in $\mathbf{C}_{L}$ are those with a recursive graph.

Proof. By Theorem 3 of [9] (which gives more than we need) there is a consistent complete extension $\mathbf{T}$ of Peano arithmetic in which the only semi-representable relations are either recursive or non-arithmetical. Define $\mathbf{C}$ as above. Then $\mathbf{C}$ has all the required properties except, perhaps, the last. For that, let $f: \mathbb{N}^{n} \rightarrow \mathbb{N}$ be a partial recursive function, and suppose that it is strongly representable in $\mathbf{C}_{L}$ by $[\gamma]: \mathrm{N}^{n} \rightarrow \mathrm{N}$. Then, for all $k_{1}, \ldots, k_{n}$, we have:

$$
f\left(k_{1}, \ldots, k_{n}\right) \simeq k \equiv[\gamma] \circ\left\langle\underline{k_{1}}, \ldots, \underline{k_{n}}\right\rangle=\underline{k} \equiv \vdash_{\mathbf{T}} \gamma\left(\left\langle\underline{k_{1}}, \ldots, \underline{k_{n}}\right\rangle, \underline{k}\right)
$$

(making use of an evident primitive recursive $n$-tupling function). So the graph of $f$ is semi-representable in $\mathbf{T}$ and is therefore recursive.

\section{Acknowledgements}

I thank Jeff Egger, Phil Scott, and Alex Simpson for very helpful discussions.

\section{References}

1. S. Alves, M. Fernández, M. Florido, and I. Mackie, Linear recursive functions, Rewriting, Computation and Proof, Essays Dedicated to Jean-Pierre Jouannaud on the Occasion of His 60th Birthday (eds. H. Comon-Lundh, C. Kirchner, and H. Kirchner), LNCS, 4600, 182-195, Springer, 2007.

2. M. Barr and C. Wells, Category Theory for Computing Science, Prentice Hall, 1998, also available as Reprints in Theory and Applications of Categories, 22, 1-538, www.tac.mta.ca/tac/reprints/, 2012.

3. A. Bucalo, C. Führmann, and A. K. Simpson, An equational notion of lifting monad, Theor. Comput. Sci. 294(1/2), 31-60, 2003.

4. J. R. B. Cockett and S. Lack, Restriction categories II: partial map classification, Theor. Comput. Sci. $\mathbf{2 9 4}(1 / 2), 61-102,2003$.

5. G. Gierz, K. H. Hofmann, K. Keimel, J. D. Lawson, M. Mislove, and D. S. Scott, Continuous Lattices and Domains, Encyclopedia of Mathematics and its Applications, 93, CUP, 2003.

6. M. Gladstone, Simplification of the recursion scheme, J. Symb. Logic, 36(4), 653-665, 1971.

7. J. Lambek and P. J. Scott, Introduction to Higher-Order Categorical Logic, Cambridge Studies in Advanced Mathematics, 7, CUP, 1988.

8. B. P. F. Jacobs, Semantics of weakening and contraction, Annals of Pure and Applied Logic, 69, 73-106, 1994.

9. C. G. Jockusch, Jr. and R. I. Soare, $\Pi_{1}^{0}$ classes and degrees of theories, Trans. Amer. Math. Soc., $\mathbf{1 7 3}(2), 33-56,1972$. 
10. P. T. Johnstone, Sketches of an Elephant: a Topos Theory Compendium, 1, OUP, 2002.

11. I. Mackie, L. Román, and S. Abramsky, An internal language for autonomous categories, Journal of Applied Categorical Structures, 1, 311-343, 1993.

12. R. Paré and L. Román, Monoidal categories with natural numbers object, Studia Logica, 48(3), 361-376, 1989.

13. A. J. Power and E. Robinson, Premonoidal categories and notions of computation, Mathematical Structures in Computer Science, 7(5), 453-468, 1997.

14. C. Smoryński, Logical Number Theory I, Springer, 1991. 